\title{
Tumor-associated methylation of the putative tumor suppressor AJAP1 gene and association between decreased AJAP1 expression and shorter survival in patients with glioma
}

\author{
David Cogdell', Woonbok Chung ${ }^{2}$, Yuexin Liu', J. Matthew McDonald ${ }^{1}$, \\ Kenneth Aldape ${ }^{1}$, Jean-Pierre J. Issa ${ }^{2}$, Gregory N. Fuller ${ }^{1}$, and Wei Zhang ${ }^{1}$
}

\section{Abstract}

Allelic loss of the short arm of chromosome 1 has been observed frequently in a wide spectrum of cancers, most frequently in oligodendroglioma. In our previous studies, we evaluated 177 oligodendroglial tumor samples and identified the AJAP1 gene (formerly Shrew1) in the consensus region of deletion. AJAP1 is a transmembrane protein found in adheren junctions and functions to inhibit glioma cell adhesion and migration. Whereas a putative tumor suppressor gene, we did not detect AJAP1 gene mutations. In subsequent studies, we found that $A J A P 1$ was underexpressed in oligodendrogliomas relative to normal brain tissues. Bioinformatic analysis revealed the presence of $\mathrm{CpG}$ islands in the promoter of $A J A P 1$. Methylation analysis of the AJAP1 promoter identified hypermethylation in $21 \%$ of oligodendrogliomas $(n=$ $27)$, and the degree of methylation correlated with low levels of $A J A P 1$ expression $(P=0.045)$. The AJAP1 promoter was also highly methylated in a wide spectrum of cell lines $(n=22)$, including cell lines of glioblastoma. Analysis of the National Cancer Institute's REMBRANDT dataset, which contains 343 glioma samples, indicated that low AJAP1 gene expression was associated with decreased survival. Thus, both genetic (gene deletion) and epigenetic alterations (promoter methylation) are likely mechanisms that inactivate the putative tumor suppressor AJAP1 in gliomas, which contributes to poor prognosis.

Key words Methylation, epigenetics, AJAP1(Shrew1), 1p36, glioblastoma, The Cancer Genome Atlas, survival

Cancer is caused by an accumulation of genetic and epigenetic alterations. Gene silencing by hypermethylation of $\mathrm{CpG}$ islands in promoter regions is a common epigenetic abnormality in cancer and may lead to a loss of function of tumor suppressor genes, such as p16, MGMT, RASSF1A, and $h M L H 1^{[1-4]}$.

Oligodendroglioma is one of the most common primary neoplasms of the central nervous system among

Authors' Affiliations: 'Department of Pathology, The University of Texas MD Anderson Cancer Center, Houston, Texas 77030, USA; '2Department of Leukemia, The University of Texas MD Anderson Cancer Center, Houston, Texas 77030, USA.

Corresponding Authors: Wei Zhang or Gregory N. Fuller, Department of Pathology, Unit 85, The University of Texas MD Anderson Cancer Center, 1515 Holcombe Blvd., Houston, Texas 77030, USA. Tel: +1-713745-1103; Fax: +1-713-792-5549; Email: gfuller@mdanderson.org or wzhang@mdanderson.org. adults in the fourth and fifth decades of life ${ }^{[5]}$. A common and characteristic chromosomal abnormalities observed in oligodendroglioma is allelic loss of $1 p^{[6-11]}$. Earlier deletion mapping studies identified most consensus deletion regions involving the $1 p 36$ region ${ }^{[8,11,12]}$. Additional mapping studies in recent years have further narrowed the consensus regions of deletion. One study with 52 oligodendrogliomas identified two regions of interest: one region contained the $D F F B$ gene, which we also found to be down-regulated in oligodendrogliomas with the $1 p / 19 q$ deletion ${ }^{[13]}$, whereas the other region contained the AJAP1 gene ${ }^{[14]}$. In our earlier studies with 177 oligodendrogliomas, we found that the second region, containing AJAP1, was in the consensus region of deletion ${ }^{[15]}$. AJAP1 is a putative tumor suppressor gene found on chromosome 1 in the 1 p36 region commonly associated with cancer ${ }^{[11]}$ and has been implicated in 
cancer cell invasion, adhesion, and migration [15,16]. Although we did not find mutation of the AJAP1 gene in oligodendrogliomas, its expression at transcript level was lower than in normal brain tissue. Immunohistochemistry using clinical samples revealed an association between AJAP1 levels and survival in patients with astrocytic glioma $^{[17]}$. Additionally, restoration of AJAP1 expression in glioma cell line inhibited adhesion and migration ${ }^{[15] .}$ Thus, AJAP1 may be a tumor suppressor whose function can be attenuated by a loss in copy number and a decrease in expression.

In this study, we investigated the possible contribution of promoter methylation to decreased AJAP1 expression in cancer cells. Analysis of an independent microarray dataset, the Repository for Molecular Brain Neoplasia Data (REMBRANDT) ${ }^{[18]}$ linked the AJAP1 expressions levels to survival.

\section{Materials and Methods}

\section{Samples and DNA isolation}

The records of 37 patients who underwent treatment for oligodendroglial tumors at The University of Texas MD Anderson Cancer Center between 1981 and 2002 were collected and reviewed with approval from the Institutional Review Board. These tumors were initially diagnosed by neuropathologists at MD Anderson as 1) low-grade oligodendroglioma or mixed oligoastrocytoma, 2) anaplastic oligodendroglioma (AO) or mixed oligoastrocytoma, or 3) glioblastoma with a significant oligodendroglial component, and the diagnoses were later confirmed by two of the authors (K.A. and G.F.). Mixed tumors were included in this study because clear pathologic discrimination between the diffuse glioma subtypes is often difficult and subjective and, as a group, oligoastrocytomas often exhibit the $1 p / 19 q$ deletion, a genetic signature that has been observed in both the oligodendroglial and astrocytic components of mixed tumors $^{[19]}$.

Tissue for DNA isolation was obtained from paraffin-embedded samples. Each tissue block was histologically assessed for tumor by a neuropathologist (K.A.). Sections were directly cut from the block for DNA isolation if at least $90 \%$ of the tissue was determined to be tumor. If the proportion of tumor was $<90 \%, 10$ to 20 unstained slides were prepared from the block, and tumor tissue was dissected from normal tissue. DNA was isolated by digesting deparaffinized tumor sections for 3 to 5 days with proteinase $\mathrm{K}$ at $55^{\circ} \mathrm{C} \quad[0.5 \mathrm{mg} / \mathrm{mL}$ in $100 \mathrm{mmol} / \mathrm{L} \mathrm{NaCl}, 10 \mathrm{mmol} / \mathrm{L}$ Tris- $\mathrm{HCl}$ ( $\mathrm{pH} \mathrm{8.0),} 25$ $\mathrm{mmol} / \mathrm{L}$ ethylenediaminetetraacetic acid, $0.5 \%$ sodium dodecyl sulfate], followed by a phenol:chloroform: isoamyl alcohol extraction and isopropanol precipitation. Tissue for RNA isolation was obtained from fresh/frozen samples. Each frozen section was histologically assessed for tumor by a neuropathologist (G.F. or K.A.) and used only if at least $90 \%$ of the tissue was determined to be tumor. For RNA isolation, up to $50 \mathrm{mg}$ of tissue was frozen in liquid nitrogen, crushed into powder using a mortar and pestle, and dissolved in $1 \mathrm{~mL}$ of Trizol reagent (Invitrogen). Then, $200 \mu \mathrm{L}$ of chloroform was added to the sample, and the sample was vortexed at high speed for $15 \mathrm{~s}$ and centrifuged at $12000 \times g$ for $15 \mathrm{~min}$ at $4^{\circ} \mathrm{C}$. After transfer of the aqueous phase to a fresh $1.5 \mathrm{~mL}$ Eppendorf tube, an equal volume of $70 \%$ ethanol was added, and the mixture was mixed by tube inversion. The sample was then loaded onto a Qiagen RNeasy column and centrifuged at $16000 \times g$ for $20 \mathrm{~s}$. The column was washed twice with $500 \mu \mathrm{L}$ of RPE buffer (Qiagen). RNA was eluated off the column in $50 \mu \mathrm{L}$ of nuclease-free water. RNA was quantified using a spectrophotometer (Nanodrop) and qualified using a 2100 BioAnalyzer (Agilent). Cell lines used in the study were CaCO2, CaMa1, HL60, 468, K562, SKHep, Hep3B, HepG2, DU145, PC3, LNCaP, UC6, UC13, SVHVC, LOVO, SW480, RKO, SW480, RS4;11, Raji, MCF7, and BT474.

\section{Quantitative reverse transcriptase-polymerase chain reaction (RT-PCR) assay for AJAPI expression}

Initial experiments were performed to determine the valid range of RNA concentrations and to demonstrate the similarity of PCR efficiencies for AJAP1 compared to the endogenous control gene cyclophilin. To determine changes in AJAP1 expression, $1 \mu \mathrm{g}$ of total RNA from samples was reverse transcribed in a $20 \mu \mathrm{L}$ reaction. To the RNA, $0.4 \mu \mathrm{g}$ of random hexamers was added, and the mixture was heated at $70{ }^{\circ} \mathrm{C}$ for $10 \mathrm{~min}$. The tubes were then incubated at room temperature for $10 \mathrm{~min}$, and the following components were added: $1 \times$ Superscript II RT buffer (Invitrogen), $10 \mathrm{mmol} / \mathrm{L}$ dithiothreitol (Invitrogen), $0.5 \mathrm{mmol} / \mathrm{L}$ deoxynucleotide triphosphate (ISC Bioexpress), $20 \mathrm{U}$ of RNase inhibitor (Ambion), and $200 \mathrm{U}$ of Superscript II RT (Invitrogen). The reaction was again incubated for $10 \mathrm{~min}$ at room temperature to allow primer annealing and then held incubated at $37^{\circ} \mathrm{C}$ for $1 \mathrm{~h}$. The reaction was spiked with an additional $200 \mathrm{U}$ of Superscript II reverse transcriptase (Invitrogen) and then incubated at $42^{\circ} \mathrm{C}$ for $90 \mathrm{~min}$ and at $50^{\circ} \mathrm{C}$ for $30 \mathrm{~min}$. Real-time PCR was performed on an $\mathrm{ABI}$ Prism 7700 using Assay-on-Demand for AJAP1 (Hs00218945_m1) and cylophilin Vic-labeled pre-developed assay reagent (4326316E) (Applied Biosystems). A $50 \mu \mathrm{L}$ final reaction volume containing $1 \times$ TaqMan Universal PCR Master 
Mix (Applied Biosystems) and $1 \times$ Assay-on-Demand (Applied Biosystems) was used to amplify $50 \mathrm{ng}$ of cDNA under the following cycling conditions: $10 \mathrm{~min}$ at $95^{\circ} \mathrm{C}$ followed by 40 cycles of $95^{\circ} \mathrm{C}$ for $15 \mathrm{~s}$ and $60^{\circ} \mathrm{C}$ for $1 \mathrm{~min}$. The $\triangle \triangle \mathrm{Ct}$ method was used to calculate fold-change in AJAP1 expression in $1 \mathrm{p}$ loss cases relative to matched $1 p$ intact cases. Calculations were also done to compare AJAP1 expression in tumors with AJAP1 expression in normal brain tissue RNA (Clontech).

\section{Methylation assay for the $A J A P 1$ promoter}

Bisulfite treatment of DNA was performed as previously described ${ }^{[15]}$. We used combined bisulfite restriction analysis (COBRA) as a quantitative test to study AJAP1 methylation in all cases ${ }^{[2,21]}$. Two separate regions in the promoter were examined and gave similar results: primers 5' - GTGTTATTYGGTTATTTTGGTAATA3' (sense) and 5' - ACСTCCTACAACCCCCTTC-3' (antisense) were used to amplify a 134-bp fragment upstream of the promoter $\mathrm{CpG}$ island, whereas primers 5'-GAGTTTTYGGGTAATTTGAGTG-3' (sense) and 5'-ACTCTTACCTCCAACCRAAAC-3' (antisense) were used to amplify a 162-bp fragment around the transcription start site. To determine the methylation status of these regions, we restricted the 134-bp fragments with BstU I and restricted the 162-bp fragments with Taq I and Dpn II. All restriction products were visualized using $6 \%$ polyacrylamide gel electrophoresis followed by staining with ethidium bromide and imaging/quantitation using a BioRad Beldoc 2000 imager (BioRad). The identities of the amplified fragments were verified using digestion with multiple restriction enzymes as well as sequencing. Setup of the PCR assays included positive and negative controls, mixing experiments to rule out bias, and repeat experiments to assess reproducibility. In this analysis, these enzymes digested only methylated alleles. In selected cases, methylation of $\mathrm{CPG}$ sites within AJAP1 was confirmed using bisulfite sequencing. The 162-bp PCR products were cloned into a TA cloning vector pCR4-TOPO (Invitrogen), and plasmid clones were extracted with a QIAprep Spin Miniprep kit (Qiagen) and sequenced at the MD Anderson Core Sequencing Facility.

\section{Bioinformatic analysis}

A Kaplan-Meier plot for gene expression data was generated using the "simple search" function within the REMBRANDT access Web site. AJAP1 was queried against all Affymetrix glioma data using the default up-regulation and down-regulation parameter of 2-fold. The AJAP1 down-regulated $(n=245$ cases $)$ and intermediately regulated ( $n=98$ cases) yielded a log-rank
$P$ value of 0.001 . AJAP1 expression and methylation data were obtained from The Cancer Genome Atlas (TCGA) public database (http://www.cancergenome. nih.gov/dataportal) as available in December 2011 ${ }^{[18]}$. Correlation between gene expression and methylation was assessed in terms of Pearson correlation, and $P$ value as determined using a two-tailed $t$-test.

\section{Results and Discussion}

Although our previous sequence analysis did not reveal mutations in the coding region of the AJAP1 (Shrew1) gene and noted the weak relationship between AJAP1 gene expression and loss of heterozygosity ${ }^{[15]}$, we next examined tumor tissues for epigenetic alterations affecting AJAP1. We theorized that a potential mechanism for the observed decreased AJAP1 expression could be promoter methylation, not only because the AJAP1 promoter contains a $\mathrm{CpG}$ island (Figure $1 \mathrm{~A}$ ) but also because promoter methylation has been shown to affect the expression of several genes in oligodendroglioma ${ }^{[22-25]}$. Thus, we theorized that AJAP1 follows the model of the p16 tumor suppressor gene, which has demonstrated a low rate of mutation in some tumor types but often shows attenuated expression due to promoter methylation ${ }^{[4]}$.

To determine if AJAP1 fits the p16 model, we compared methylation using bisulfite/restriction analysis of the promoter region in 27 oligodendroglioma samples (Figure 1B, Figure 2). In our dataset, as there were no cases with methylation levels between $16 \%$ and $20 \%$, any value in that interval was a natural choice for the threshold. Using this threshold, we detected hypermethylation in $6(21 \%)$ of the 27 cases (Figure $3 A$ ). Of interest, 4 of the 6 cases with hypermethylation had $1 p$ allelic loss. DNA methylation of the AJAP1 CpG island was frequently found in cell lines from various tissue types (19 of 22 examined) and was rare in normal tissues. DNA methylation at the proximal promoter region (162-bp) of the AJAP1 gene was confirmed in selected cases via direct bisulfite sequencing (Figure 1C).

To test the hypothesis that a high average methylation ratio was associated with decreased AJAP1 expression, we split the cases into two groups corresponding to high and low methylation. Additionally, we subjected the methylation data to an unpaired $t$-test with two clusters, which resulted in a $P$ value $<0.05$ (Figure 3A). To further verify this correlation, we obtained AJAP1 gene expression and methylation data of 253 patients from the Cancer Genome Atlas database. The data showed two methylation sites located at chr1:4613868 and chr1:4615380, named $\mathrm{M}-4613868$ and M-4615380. Distribution of gene 


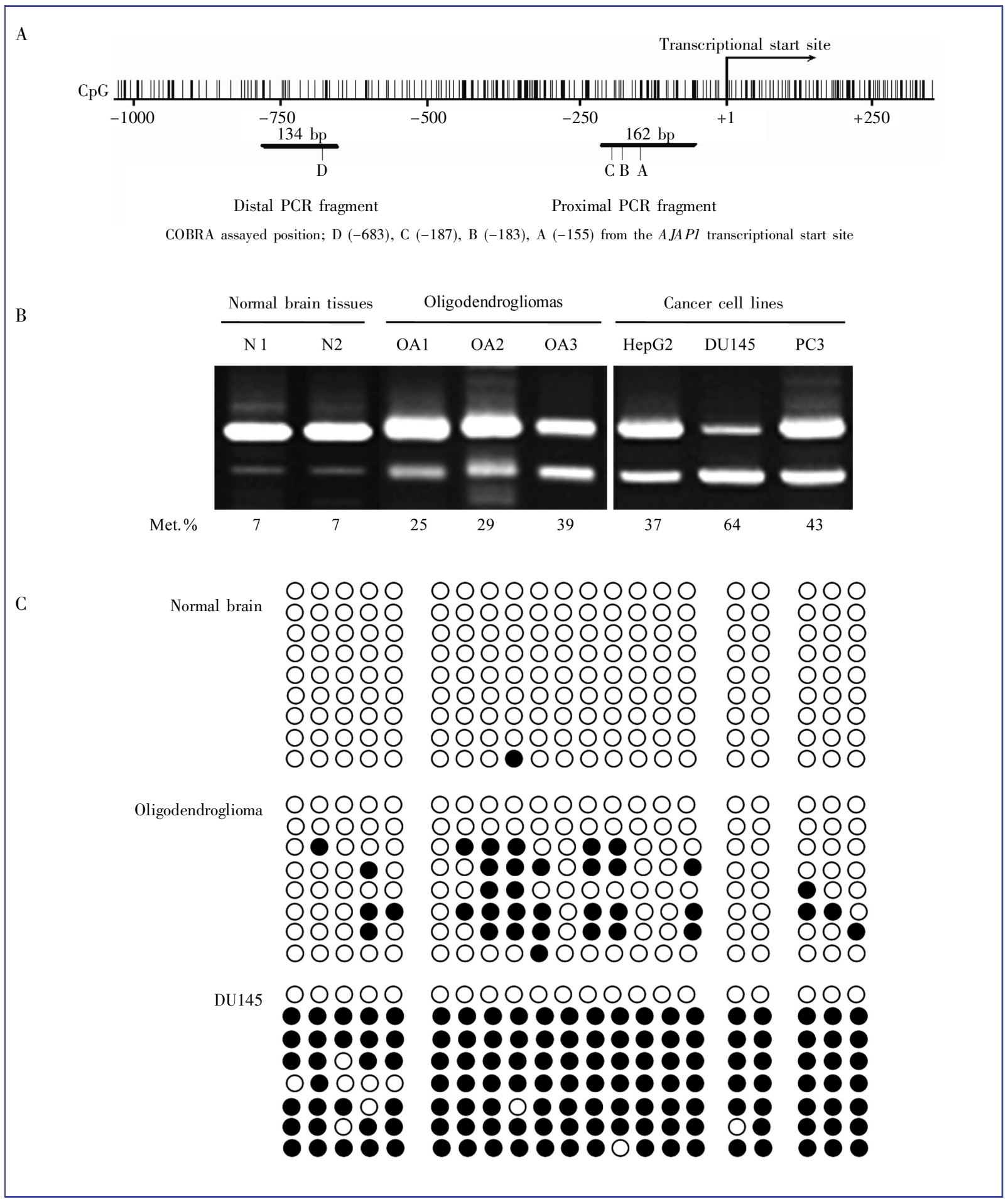

Figure 1. $\mathrm{CpG}$ map of the AJAP1 promoter, COBRA and direct bisulfate sequencing for DNA methylation analysis. A, each vertical bar represents a single $\mathrm{CpG}$ site, and the high density of $\mathrm{CpG}$ sites indicates the presence of a $\mathrm{CpG}$ island. The transcription start site is indicated by an arrow. The 162-bp proximal PCR fragment and distal 134-bp fragment are shown with the methylation-sensitive restriction enzyme sites listed. B, an example of COBRA assay at 183-bp upstream from the transcriptional start site. The PCR product was digested with Dpn II that distinguished methylated DNA from unmethylated DNA after bisulfite treatment for COBRA assay. Oligodendrogliomas (OA1, OA2, and OA3) and cancer cell lines (HepG2, DU145, and PC3) had higher levels of DNA methylation than normal brain tissues (N1 and N2). C, direct bisulfite sequencing results of normal brain (N1), oligodendroglioma (OA3), and cancer cell line DU145 of proximal 162-bp PCR fragment. Black circles, methylated CpG sites; open circles, unmethylated CpG sites. 


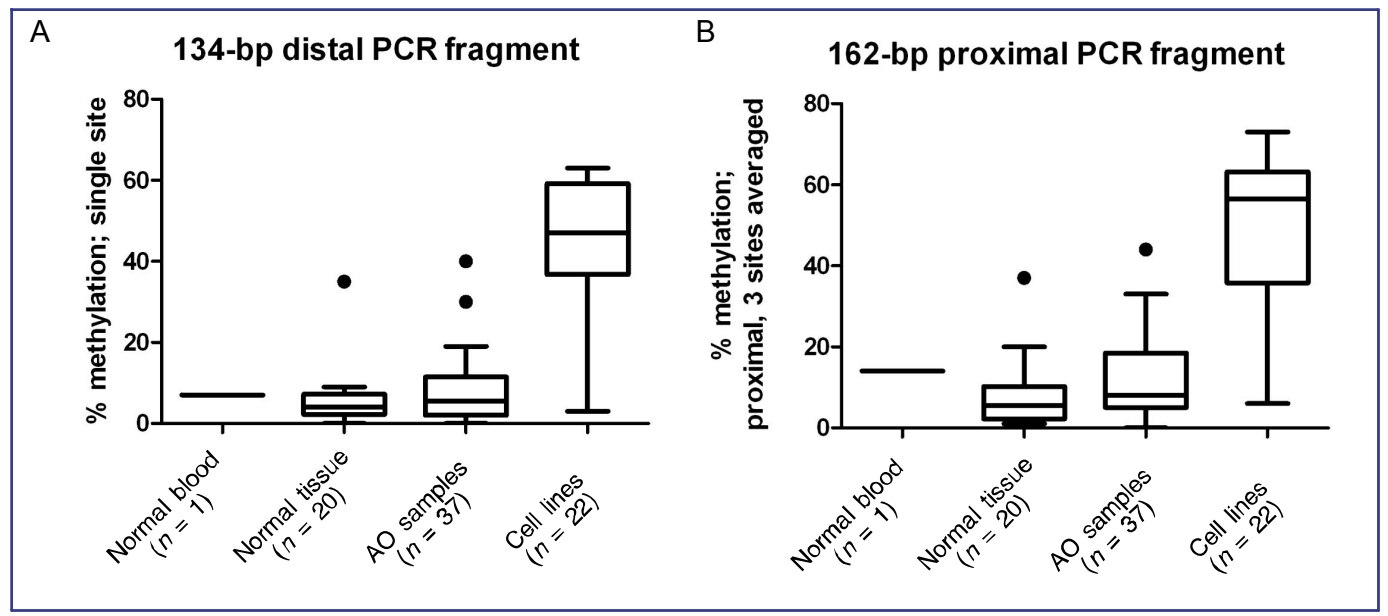

Figure 2. AJAP1 methylation of 1 sample of normal blood, 20 samples of normal tissue, 37 samples of anaplastic oligodendroglioma ( $\mathrm{AO}$ ), and 22 cell lines. The COBRA data shown with Tukey whiskers summarizing the methylation sensitive restriction enzyme data on PCR fragments in various sample types. A, distal PCR fragment. $B$, proximal $P C R$ fragment.

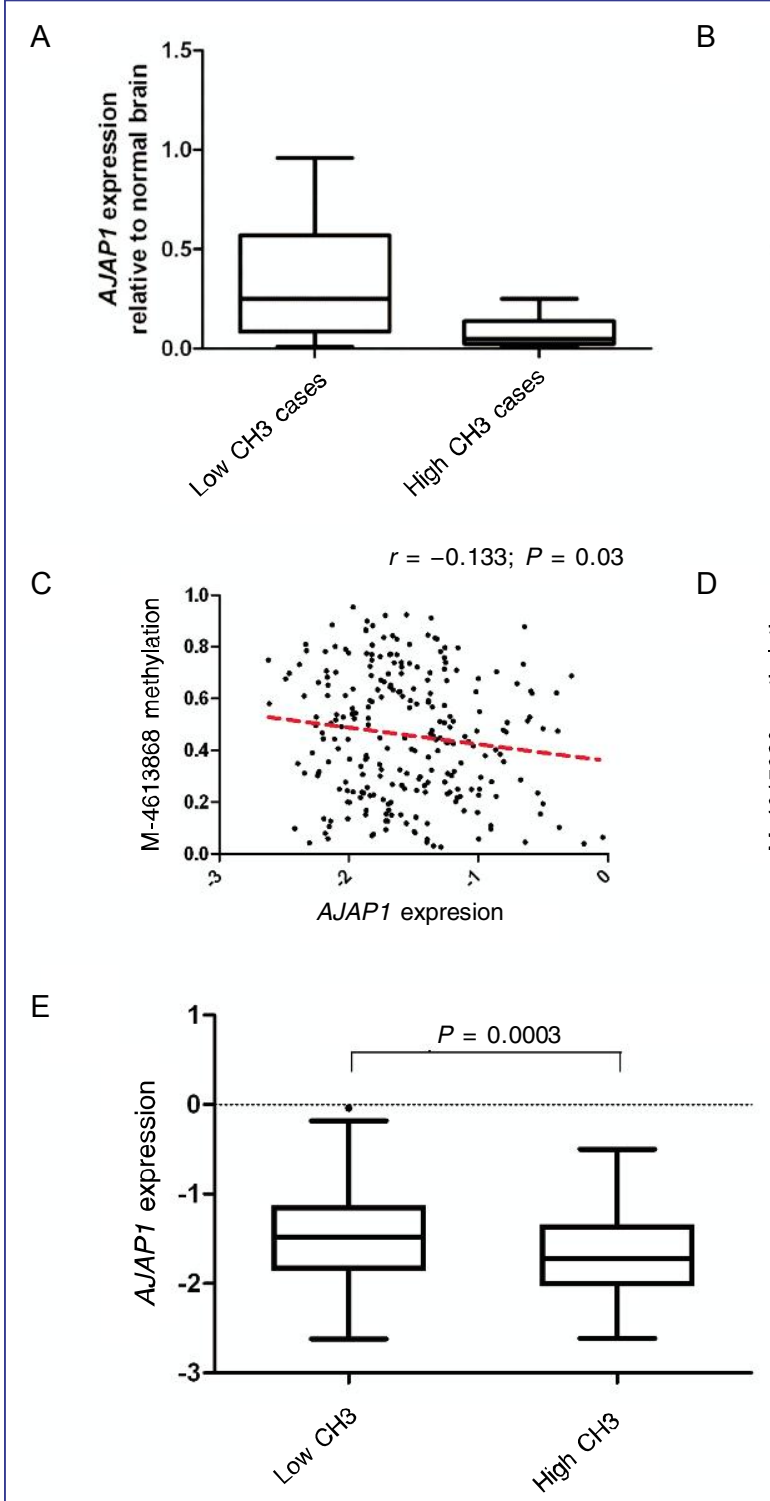

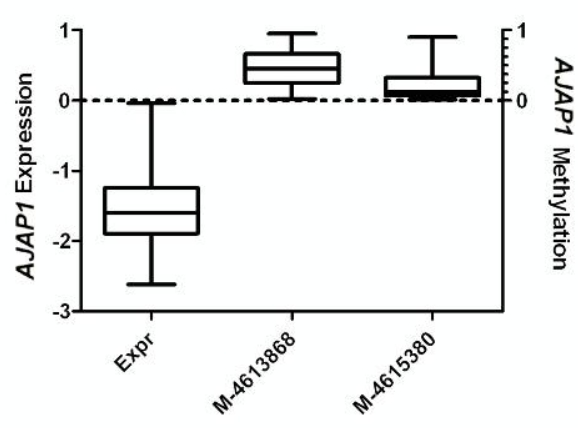

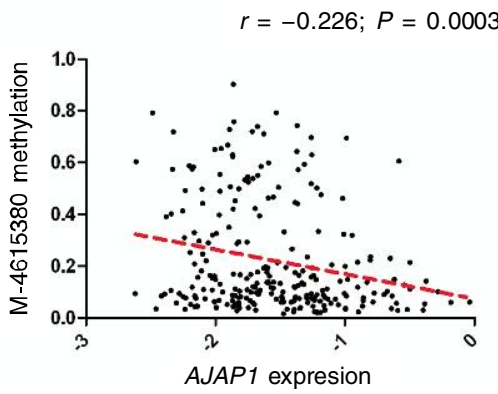

Figure 3. Correlation between methylation and AJAP1 expression. A, AJAP1 expression in 35 brain tumors was stratified into four groups according to the average methylation status of the cases. The 6 cases in the group with $>15 \%$ methylation were considered to be in the high-methylation category and had the lowest level of expression. B, distribution of AJAP1 expression and methylation across the 253 glioblastoma samples in the Cancer Genome Atlas database. C, correlation of AJAP1 expression with methylation at M-4613868. D, correlation of AJAP1 expression with methylation at M- 4615380 . The dashed red lines denote the linear regression of correlation; $r$ : Pearson coefficient. E, AJAP1 expression in the low-CH3 and high- $\mathrm{CH} 3$ groups based on the methylation values at M-4615380 using the same $\mathrm{CH} 3$ cutoff of $15 \%$. $\mathrm{CH} 3$ stands for methylation. 
expression and methylation is depicted in Figure 3B, which shows that AJAP1 was down-regulated in the samples with a median value of about -1.60 ( $\log 2$ ratio). Methylation was almost three times higher at M-4613868 $(45.6 \%)$ than at M-4615380 (12.6\%). Both M-4613868 (r $=-0.133, P=0.03)$ and $M-4615380 \quad(r=-0.226, P<$ 0.001) exhibited an inverse correlation with AJAP1 expression (Figure $3 \mathrm{C}$ and $3 \mathrm{D}$ ). In a manner similar to the one depicted in Figure $3 \mathrm{~A}$, we split the patients into two groups (144 with low methylation and 109 with high methylation) based on their values at M-4615380 using the same cutoff of $15 \%$. Consistently, patients in the low CH3 group (median -1.479) exhibited higher AJAP1 expression than those in the high $\mathrm{CH} 3$ group (median $-1.718)$ with statistical significance $(P<0.001)$ (Figure $3 \mathrm{E})$. Methylation at M-4613868 was comparatively high, and it was difficult to split the patients into two distinct groups based on the same $\mathrm{CH} 3$ cutoff. In all, we experimentally and computationally observed a statistically significant correlation between methylation of the AJAP1 promoter and AJAP1 expression. Using an independent dataset of 343 glioma samples profiled in the REMBRANDT application (http://rembrandt.nci.nih.gov) ${ }^{[26]}$, we determined the association between AJAP1 expression and survival (Figure 4). This relationship indicates that the AJAP1 gene product affects glioma progression ${ }^{[18]}$.

Thus, we propose that AJAP1 is a putative tumor suppressor gene located on $1 \mathrm{p} 36$ and inhibits cell adhesion and migration. Although no tumor-specific mutations were observed, AJAP1 may still fit the two-hit hypothesis for tumor suppressor genes in that expression from two wild-type alleles is abrogated by either gene deletion or promoter hypermethylation, or in the cases where gene deletion is absent, both hits can be manifested by promoter hypermethylation. Further investigations are needed to determine the role of AJAP1 in the etiology of oligodendroglioma and other tumor types with nonrandom 1p36 allelic loss.

\section{Acknowledgments}

The work presented in this article was partially supported by the Anthony Bullock III Research Fund and Cancer Center Support Grant CA016672 from the National Institutes of Health to the MD Anderson Cancer

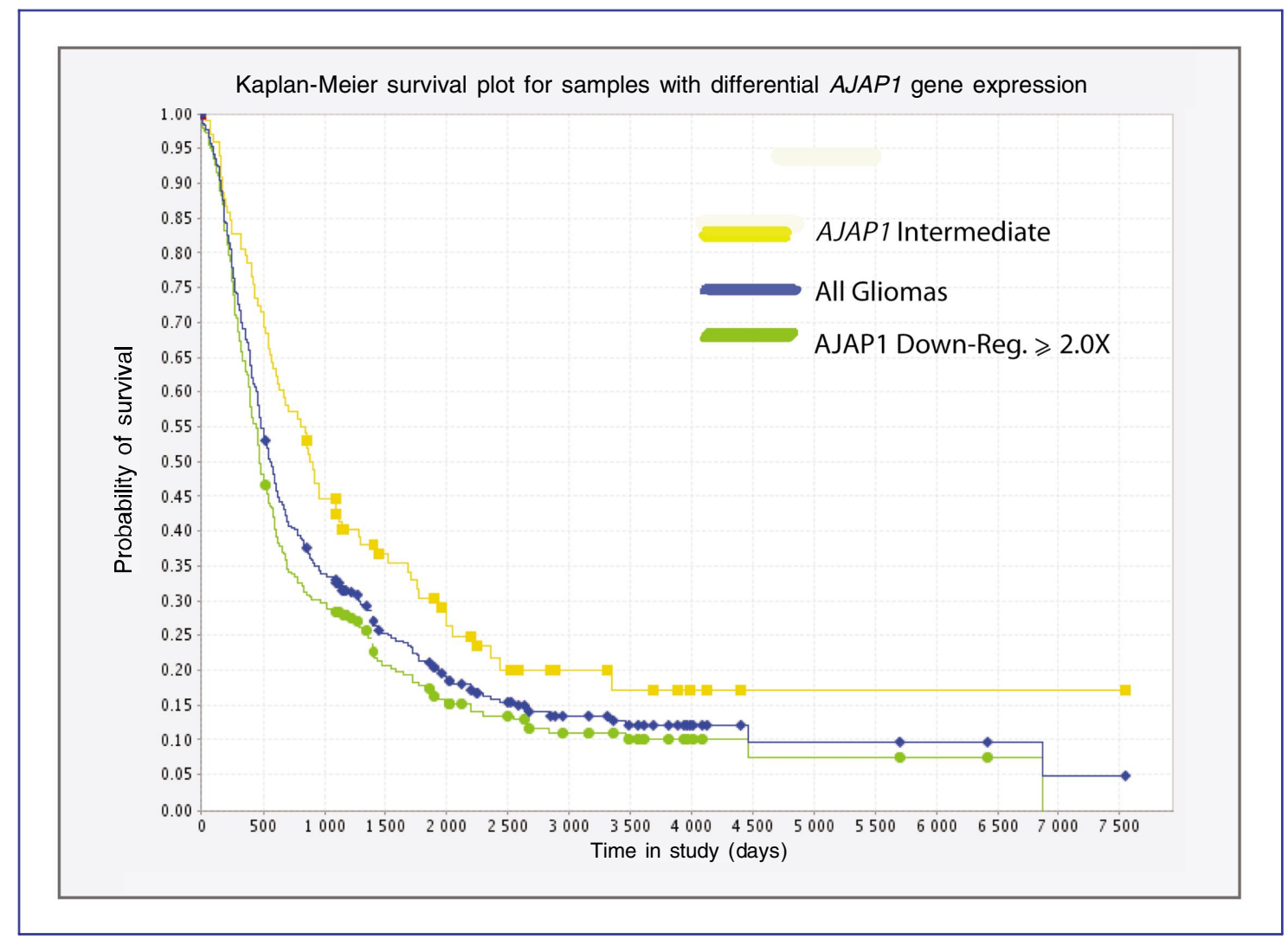

Figure 4. Association of AJAP1 expression with survival using glioma data from REMBRANDT. Of the 343 patients with glioma, 245 had down-regulated AJAP1 expression and 98 had up-regulated expression. The survival rate, calculated with the Kaplan-Meier method, was significantly lower in the patients with down-regulated AJAP1 expression than in those with intermediate expression $(P=0.001)$. 
Center. We acknowledge the Repository for Molecular Brain Neoplasia Database (REMBRANDT), created and maintained by the NINDS, NCl, at the $\mathrm{NIH}$, for the AJAP1 gene expression and survivability data. We also thank Valerie Dunmire for her technical assistance and Markeda Wade at the Department of Scientific

\section{References}

[1] Dammann R, Li C, Yoon JH, et al. Epigenetic inactivation of a ras association domain family protein from the lung tumour suppressor locus 3p21.3 [J]. Nat Genet, 2000,25(3):315-319.

[2] Esteller M, Toyota M, Sanchez-Cespedes M, et al. Inactivation of the DNA repair gene O-6-methylguanine-DNA methyltransferase by promoter hypermethylation is associated with $\mathrm{G}$ to $\mathrm{A}$ mutations in $\mathrm{K}$-ras in colorectal tumorigenesis [ $\mathrm{J}$ ]. Cancer Res, 2000,60(9):2368-2371.

[3] Herman JG, Umar A, Polyak K, et al. Incidence and functional consequences of hMLH1 promoter hypermethylation in colorectal carcinoma [J]. Proc Natl Acad Sci U S A, 1998,95 (12):6870-6875

[4] Merlo A, Herman JG, Mao L, et al. 5' CpG island methylation is associated with transcriptional silencing of the tumour suppressor p16/CDKN2/MTS1 in human cancers [J]. Nat Med, 1995, 1(7):686-692.

[5] Surawicz TS, McCarthy BJ, Kupelian V, et al. Descriptive epidemiology of primary brain and CNS tumors: results from the central brain tumor registry of the United States, $1990-$ 1994 [J]. Neuro Oncol, 1999,1(1):14-25.

[6] Bello MJ, Leone PE, Vaquero J, et al. Allelic loss at $1 p$ and 19q frequently occurs in association and may represent early oncogenic events in oligodendroglial tumors [J]. Int J Cancer, 1995,64(3):207-210.

[7] Bello MJ, Vaquero J, de Campos JM, et al. Molecular analysis of chromosome 1 abnormalities in human gliomas reveals frequent loss of $1 \mathrm{p}$ in oligodendroglial tumors [ $\mathrm{J}]$. Int $\mathrm{J}$ Cancer, 1994,57(2): 172-175.

[8] Husemann K, Wolter M, Buschges R, et al. Identification of two distinct deleted regions on the short arm of chromosome 1 and rare mutation of the CDKN2C gene from 1 p32 in oligodendroglial tumors [J]. J Neuropathol Exp Neurol, 1999,58(10):1041-1050.

[9] Nigro JM, Takahashi MA, Ginzinger DG, et al. Detection of $1 \mathrm{p}$ and $19 \mathrm{q}$ loss in oligodendroglioma by quantitative microsatellite analysis, a real-time quantitative polymerase chain reaction assay [J]. Am J Pathol, 2001,158(4):1253-1262.

[10] Reifenberger J, Reifenberger G, Liu L, et al. Molecular genetic analysis of oligodendroglial tumors shows preferential allelic deletions on 19q and 1p [J]. Am J Pathol, 1994,145(5):11751190.

[11] Smith JS, Alderete B, Minn $Y$, et al. Localization of common deletion regions on $1 p$ and $19 q$ in human gliomas and their association with histological subtype [J]. Oncogene, 1999,18 (28):4144-4152

[12] luchi $T$, Namba $H$, Iwadate $Y$, et al. Identification of the small interstitial deletion at chromosome band 1p34-p35 and its association with poor outcome in oligodendroglial tumors [J]. Genes Chromosomes Cancer, 2002,35(2):170-175.

[13] McDonald JM, Dunmire V, Taylor E, et al. Attenuated expression of DFFB is a hallmark of oligodendrogliomas with

\section{Publications of MD Anderson Cancer Center for her editorial assistance.}

Received: 2011-01-26; revised: 2011-02-25; accepted: 2011-02-25. 1p-allelic loss [J]. Mol Cancer, 2005,4:35.

[14] Dong Z, Pang JS, $\mathrm{Ng} \mathrm{MH}$, et al. Identification of two contiguous minimally deleted regions on chromosome 1p36.31-p36.32 in oligodendroglial tumours [J]. Br J Cancer, 2004,91(6):1105-1111.

[15] McDonald JM, Dunlap S, Cogdell D, et al. The SHREW1 gene, frequently deleted in oligodendrogliomas, functions to inhibit cell adhesion and migration [J]. Cancer Biol Ther, 2006,5(3): 300-304.

[16] Schreiner A, Ruonala M, Jakob V, et al. Junction protein shrew-1 influences cell invasion and interacts with invasionpromoting protein CD147 [J]. Mol Biol Cell, 2007,18(4):1272 1281.

[17] Ernst A, Hofmann S, Ahmadi R, et al. Genomic and expression profiling of glioblastoma stem cell-like spheroid cultures identifies novel tumor-relevant genes associated with survival [J]. Clin Cancer Res, 2009,15(21):6541-6550.

[18] REMBRANDT. National cancer institute. Rembrandt home page. http://rembrandt.nci.nih.gov. 2005. Accessed 2010 December 21.

[19] Kraus JA, Koopmann J, Kaskel P, et al. Shared allelic losses on chromosomes $1 p$ and $19 q$ suggest a common origin of oligodendroglioma and oligoastrocytoma [J]. J Neuropathol Exp Neurol, 1995,54(1):91-95.

[20] Cottrell SE, Laird PW. Sensitive detection of DNA methylation [J]. Ann N Y Acad Sci, 2003,983:120-130

[21] Chung W, Kwabi-Addo B, Ittmann $M$, et al. Identification of novel tumor markers in prostate, colon and breast cancer by unbiased methylation profiling [J]. PLoS One, 2008,3(4):e2079.

[22] Dong SM, Pang JC, Poon WS, et al. Concurrent hypermethylation of multiple genes is associated with grade of oligodendroglial tumors [J]. J Neuropathol Exp Neurol, 2001,60 (8):808-816.

[23] Hong C, Bollen AW, Costello JF. The contribution of genetic and epigenetic mechanisms to gene silencing in oligodendrogliomas [J]. Cancer Res, 2003,63(22):7600-7605.

[24] Watanabe $T$, Nakamura $M$, Yonekawa $Y$, et al. Promoter hypermethylation and homozygous deletion of the p14ARF and p16INK4a genes in oligodendrogliomas [J]. Acta Neuropathol, 2001,101(3): 185-189

[25] Wolter M, Reifenberger J, Blaschke B, et al. Oligodendroglial tumors frequently demonstrate hypermethylation of the CDKN2A (MTS1, p16INK4a), p14ARF, and CDKN2B (MTS2, p15INK4b) tumor suppressor genes [J]. J Neuropathol Exp Neurol, 2001,60(12): 1170-1180.

[26] Madhavan S, Zenklusen JC, Kotliarov $Y$, et al. Rembrandt: helping personalized medicine become a reality through integrative translational research [J]. Mol Cancer Res, 2009,7 (2): 157-167. 